\title{
La tarea actual de los internistas
}

Pérez. Miranda M. La tarea actual de los internistas. An Med Interna (Madrid) 2006; 23: 403-405.

Nadie pone en duda el sobresaliente papel que ha desempeñado la Medicina Interna (MI) desde su aparición, a finales del XIX, hasta la actualidad, como faro orientador, guía y centro de referencia de la asistencia médica científica. Gracias a la clarividencia, el pensamiento, el esfuerzo y la tenacidad de grandes médicos como Strümpell, Von Mering, Khrell, Klemperer, Osler y otros grandes y brillantes sucesores, nació la MI con una predominante vocación científica: con el decidido e irrenunciable propósito de incorporar a la práctica clínica los crecientes y decisivos hallazgos de la fisiología, la bioquímica, la microbiología; de la patología celular, estructural y ultraestructural y de la radiología, la inmunología, la electrofisiología y otras disciplinas científicas emergentes (1-4). Podemos afirmar, con contundencia, que la medicina científica y técnica actual ha sido posible gracias fundamentalmente a la existencia de la MI, tronco y cuna de las brillantes especialidades médicas (EM), que no hubieran existido sin ella. Por este motivo, la MI ha gozado en los últimos cien años de una aureola intelectual y de un prestigio científico, en la vanguardia del conocimiento médico, totalmente justificado e inigualable.

No obstante, la creciente multiplicación y el progresivo auge de las EM y el notable desarrollo de su protagonismo asistencial, cada vez mayor, parecen haber mermado el papel, el prestigio asistencial y la consideración que tienen de la MI tanto los propios usuarios de las instituciones sanitarias como los gestores de las mismas. Esta perdida de imagen ha generado en los internistas un cierto desánimo y una creciente insatisfacción en el desempeño de su función asistencial. Papel asistencial que ha ido quedando progresivamente desdibujado, especialmente en el ámbito de nuestro Sistema Nacional de Salud, paradójicamente a la vez que se ha ido haciendo más complejo y disperso. El Real Decreto 521/1987, sobre Estructura, Organización y Funcionamiento de los Hospitales de nuestro sistema sanitario quiso negar el papel rector de la Medicina Interna en la estructura hospitalaria al declarar como "a extinguir" las jefaturas de departamento correspondientes, en donde estaban integradas, hasta entonces, en completa conformidad con las auténticas necesidades asistenciales, las diversas especialidades médicas, y en donde éstas se desarrollaban adecuadamente, de manera armónica y coordinada. De la misma manera, el citado decreto desmembraba también de los servicios de Medicina Interna a las secciones y unidades asistenciales de las diversas especialidades médicas, haciéndolas depender directa y exclusivamente de la dirección médica del hospital, obviamente más preparada para abordar aspectos administrativos y cuantitativos, que para velar por las cuestiones conceptuales y cualitativas que deben regir las interrelaciones científicas, formativas y asistenciales naturalmente existentes entre la MI y las EM.

Por todas las razones apuntadas, es lógico que en las últimas décadas se hable con frecuencia de una situación de crisis en la MI, que ha sido abordada y discutida reiteradamente en revistas médicas y en los congresos de esta disciplina en nuestro entorno propio (5-9). La crisis no se limita al interior de nuestras fronteras, aunque es cierto que la insatisfacción y las negativas perspectivas asistenciales y formativas de la MI son mucho menores en los países anglosajones que en el nuestro (10-14). Aunque el panorama es todavía algo sombrío, resulta estimulante y esperanzador que los propios internistas estén demostrando en los últimos años un decidido propósito de mejorar su autoestima y su satisfacción profesional analizando y publicando reiteradamente la intensa labor asistencial que ellos vienen desarrollando en nuestros hospitales, tarea que sigue siendo imprescindible e insustituible, como demuestran los datos aportados en sus publicaciones (15-22).

A este tipo de estudios pertenecen los trabajos de Rayego Rodríguez (23) y de Cinza Sanjurjo (24). En el primero de ellos (23) se hace un meticuloso análisis de la mortalidad hospitalaria en un servicio de MI de un hospital de primer nivel. Este análisis se revela como un adecuado índice para evaluar la calidad hospitalaria y además permite corroborar el importante peso asistencial que sigue teniendo la MI en el sistema sanitario actual. En el segundo estudio (24), complementario y ampliamente coincidente con el anterior, queda nuevamente evidenciado el protagonismo asistencial sobresaliente que la MI continúa teniendo también en un hospital universitario de referencia, respecto a las patologías más prevalentes. En ambos estudios queda patente que la forma- 
ción integral y los sólidos conocimientos que caracterizan a los internistas les habilitan como los más idóneos para afrontar la compleja co-morbilidad de una sociedad que tiene una progresiva prolongación de su esperanza de vida y cuyos rápidos y notables cambios demográficos y sociales constituyen un auténtico desafío para el sistema sanitario. El internista, que sigue siendo leal y fiel a la vocación científica originaria de los fundadores y primeros maestros de la MI, por su sólida formación integral e integradora y por su vocación humanística y humanitaria, sigue estando situado en la cumbre de la actividad asistencial hospitalaria, como demuestran los estudios referidos. La demanda asistencial generada por una creciente pluripatología obliga a la MI a seguir aportando sus conocimientos biológicos y fisiopatológicos, su larga experiencia en la valoración clínica global y su inquebrantable vocación por la asistencia médica integral científicamente fundamentada (23).

Todas estas realidades, que demuestran que la MI sigue desempeñando un papel central y fundamental en la asistencia médica, han sido analizadas meticulosamente por el magnífico estudio encargado por la SEMI a $S \& H$ Medical Science Service que ha sido publicado recientemente (24). En dicho estudio, que merece una larga y reposada lectura, una reflexión profunda por parte de todos los internistas y una atención especial por parte de los gestores de la sanidad pública, se demuestra con solidez la enorme carga asistencial que soportan los internistas, responsables de la asistencia de los 10 GRD más frecuentes del hospital, compartiendo un $70 \%$ de esta gran carga asistencial con todas las otras EM más específicas, lo que confirma la polivalencia y versatilidad de los internistas. Los procesos que más frecuentemente atienden los internistas, exclusiva o predominantemente, son las afecciones cardio- y cerebro- vasculares, las patologías infecciosas, metabólicas y degenerativas y las relacionadas con hábitos tóxicos y causas medioambientales. No eluden tampoco una presencia sobresaliente en la asistencia a patologías digestivas, anemias y otros trastornos hematológicos.

Un aspecto nada despreciable del trabajo hospitalario realizado por el internista es el hecho de que el coste medio de los procesos médicos atendidos por él es un 12,6\% inferior al promedio de los atendidos por las restantes especialidades médicas del hospital y el más bajo de todos ellos. En la costosa medicina tecnificada actual este es un dato que no deben despreciar los gestores sanitarios. La eficacia asistencial de la MI es igualmente indudable ya que gestiona ella sola el 14,8\% de las altas hospitalarias, frente al 15,9\% de las altas hospitalarias gestionadas por todas las demás EM conjuntamente. Por otra parte, la atención en consultas externas de la compleja casuística pluripatológica que es propia de la MI tiene un costo medio de 483 euros por proceso, prácticamente similar al costo de la casuística, mucho más puntual y concreta, de las diversas EM, cuyo promedio global es de 465 euros por proceso.

Si estos datos cuantitativos tienen un significado claramente indicativo de la vigencia que sigue teniendo el papel hospitalario central que era tradicional en la MI, los aspectos cualitativos de la actividad profesional que sigue desarrollando día a día son aún más significativos y convincentes. El Working Group on Political Issues in Internal Medicine in Europe ha definido a la MI como "la disciplina médica principal en el cuidado de los adultos con una o varias enfermedades agudas o crónicas complejas que incluye un cuidado multisistémico e integra a otras especialidades médicas". $\mathrm{Su}$ actividad está centrada en el paciente, dando una atención basada en principios éticos, científicos y holísticos. El médico internista tiene una visión de conjunto del paciente y es capaz de orientar cualquier patología médica. Cuida a enfermos que fundamentalmente padecen procesos que afectan a más de un órgano o sistema. Es el especialista con mayor capacidad para conocer el ámbito del resto de las especialidades.

Hay que mencionar, para finalizar, que junto a la vigencia actual del papel tradicional del internista su figura se ha visto agrandada al asumir nuevas funciones que se están imponiendo progresivamente en los modernos sistemas de atención socio-sanitaria: cuidados paliativos, asistencia extrahospitalaria, consultas de alta resolución, coordinación y atención de urgencias, atención de pacientes ancianos pluripatológicos, gestión de procesos médicos y consultoría de medicina interna en atención primaria.

\section{PÉREZ MIRANDA}

Departamento de Medicina Interna. Hospital Universitario Infanta Cristina. Badajoz

\section{Bibliografía}

1. Krehl L. Handbuch der inneren Medizin. Halle, 1901. Ed española en 1921.

2. Bean WB. Origin of the term Internal Medicine. N Engl J Med 1982; $306(3): 82-3$.

3. Strümpell A. Lehrbuch der speziellen Pathologie und Therapie der inneren Krankheiten. Leipzig 1883 (Edicion española en 1885).

4. Rico Irlés J. ¿Réquiem por la Medicina Interna? An Med Interna (Madrid) 2004; 21: 521-522.

5. Rozman C. Reflexiones sobre la Medicina Interna en la Universidad.
Med Clin (Barc) 1981; 76: 193-202.

6. Lissen E. El Departamento de Medicina Interna en las Ciudades Sanitarias de la Seguridad Social. Med Clin (Barc) 1983; 80: 167-171.

7. Ortiz Vázquez J. Presente y futuro de la Medicina Interna como especialidad, como departamento hospitalario y como enseñanza. An Med Interna (Madrid) 1984; 1: 100-107

8. Asenjo Sebastián, MA. El Servicio de Medicina Interna en la Organización hospitalaria. Libro de actas del XVIII Congreso de la Sociedad Española de Medicina Interna. 1988; Madrid, 207-226. 
9. Lissen E. La medicina interna general a debate: historia de una crisis y su recuperación. Med Clin (Barc) 1995; 105: 142-147

10. López Santolito A. Formación de internistas en Estados Unidos. Libro de actas del XVIII Congreso de la Sociedad Española de Medicina Interna. Madrid; 1988. p. 29-31.

11. American College of Physicians. The role of the future General Internis defined. Ann Intern Med 1994; 121: 616-622.

12. Haas JS, Cook EF, Puopolo AL, Burstin HR, Cleary PD, Brennan TA. Is the professional satisfaction of general internists associated with patient satisfaction? J Gen Intern Med 2000; 15: 122-128.

13. Pathman DE, Konrad TR, Williams ES, Scheckler WE, Linzer M, Douglas J. Physician job satisfaction, dissatisfaction, and turnover. J Fam Pract 2002; 51: 593-593.

14. Abigail Z. Dissatisfaction with Medical Practice. N Engl J Med 2004; 350: 69-75

15. Suárez Herranz P, Gabarró López N, Gil Gómez J, Millán Núñez-Cortés J. Evaluación de la calidad asistencial en la insuficiencia cardiaca. An Med Interna (Madrid) 1999; 16: 553-556.

16. Encuesta Nacional de la Sociedad Española de Medicina Interna sobre insuficiencia cardiaca. Rev Clin Esp 2001; 201 (Supl. 1): 3-6.

17. Grupo de trabajo de insuficiencia cardiaca de la Sociedad Española de Medicina Interna (SEMI). La insuficiencia cardiaca en los servicios de medicina interna (estudio SEMI-IC). Med Clin (Barc) 2002; 118: 60510.

18. García Lázaro M, Lucena Merino MM, Montero Pérez-Barquero M, Sánchez Guijo P. Estudio de la actividad asistencial de la Medicina Interna en relación a las interconsultas. An. Med. Interna (Madrid) 2003, 20: 510-14.
19. Navarro Sanz R. ¿Es la atención sanitaria a las personas mayores con enfermedad avanzada una alternativa asistencial de la Medicina Interna? An Med Interna (Madrid) 2004; 21: 315-316.

20. Sanclemente C, Barcons M, Moleiro MA, Alonso F, Pañella D, Carrera $\mathrm{R}$, et al. Mortalidad hospitalaria en un Servicio de Medicina Interna. An Med Interna (Madrid) 2004; 21: 317-321.

21. Delgado Morales JL, Alonso del Busto R, Pascual Calleja I, Villacorta Martín MM, Ergueta Martín P, González Sarmiento E. Estudio observacional de los pacientes ingresados en un Servicio de Medicina Interna. An Med Interna (Madrid) 2004; 21: 3-6.

22. Álvarez Frías MT, Gutiérrez Dubois J, Alonso Martinez JL, Solano Remírez M, González Arencibia C, Etxegaray Agara M. Calidad del manejo de pacientes con insuficiencia cardiaca en el Servicio de Medicina Interna. An Med Interna (Madrid) 2005; 22: 309-12.

23. Rayego Rodríguez J, Rodríguez-Vidigal FF, Mayoral Martín L, Álvarez-Oliva A, Najarro Díez F. Mortalidad hospitalaria en el Servicio de Medicina Interna de un hospitl de primer nivel. An Med Interna (Madrid) 2006; 23: 406-10.

24. Cinza Sanjurjo S, Cabarcos Ortiz de Barrón A, Nieto Pol E, Lorenzo Zúñiga V. Análisis epidemiológico de los pacientes ingresados en un Servicio de Medicina Interna. An Med Interna (Madrid) 2006; 23: 41115.

25. De Portugal Álvarez J. Conocimientos y saber en Medicina. An Med Interna (Madrid) 2004; 21: 1-2.

26. SEMI. Del Campo Martín, A. (Dir: S \& H Medical Science Service) Estudio socioprofesional de la Medicina Interna en España. Prospectiva 2010. (Ed. Novartis Farmacéutica S.A., Marzo 2005). http://www.fesemi.org/publicaciones 